\title{
Mid-frequency band gap performance of sandwich composites with unconventional core geometries
}

\author{
T. Ampatzidis*a , D. Chronopoulos ${ }^{\mathrm{a}}$ \\ ${ }^{a}$ Institute for Aerospace Technology \& The Composites Group, University of Nottingham, \\ $N G 81 B B, U K$
}

\begin{abstract}
In this work novel unconventional core architectures are presented which are able to induce flexural band gaps while not being detrimental for structural bending stiffness of the sandwich structures. Two different core schemes are examined with both of them exhibiting low-frequency stop bands. While unconventional, the designs of the core offer a novel solution which can be easily manufactured in high volume parts using two-dimensional automated cutting machine. A hybrid finite element and periodic structure theory scheme is employed for the calculation of the stiffness and mass matrices, and periodic structure theory is used to obtain the wave propagation of the beams. Having acquired the wave dispersion curves and the finite element analysis' results, two specimens are manufactured using carbon fibre cured plates and commercially available PVC foam as core material. Experimental measurements of the dynamic performance of the structures are conducted using a laser vibrometer and electrodynamic shaker setup.
\end{abstract}

Keywords: Sandwich Structure, Composite Materials, Wave Stop-Band, Wave Propagation, Wave Finite Elements Method

Email address: Theofanis.Ampatzidis@nottingham.ac.uk (T. Ampatzidis*) 


\section{Introduction}

One of the main issues that transport vehicles' industry faces is the noise and vibration transmission within payload and passenger compartment. To achieve high standards of their products, manufacturers need to optimise simultaneously 5 the mechanical and the vibroacoustic performance of structural assemblies. It has been demonstrated that judiciously designed periodic structures can induce vibration attenuation and stop-band behaviour in specific frequency ranges (socalled band gaps or stop bands).

In 19th century mathematical tools were developed which later would be used to calculate periodic structures' behaviour. In Floquet's mathematical work [1] the one-dimensional (1D) Mathieu's equations were studied as part of the theory of differential equations with periodic coefficients. Floquet's work was followed by that of Rayleigh [2], who developed a similar form to Floquet's theorem, which transforms the periodic system to a linear system with con-

15 stant, real coefficients. During the twentieth century and based on the existing mathematical tools, Mead [3, 4], Mace et al. [5] and Langley and Cotoni et al. $[6,7]$ produced mathematical tools based on Brillouin's periodic structure theory (PST) [8]. Using these methods, researchers nowadays have the ability to predict the vibroacoustic and dynamic performance of several applications in relatively short times. Application examples are presented using composite panels and shells $[9,10]$, structures with pressurised shells [11], and complex periodic structures $[12,13,14,15]$.

The two major mechanisms that have been identified to generate band gap behaviour in periodic structures are the Bragg scattering and local resonance.

25 Bragg scattering is observed when a structure exhibits periodic impedance mismatches and the waves are scattered at the borders of the unit-cell (the part of the structure that is periodically repeated). This scattering can be caused by means of inclusions, and geometrical or material inconsistencies. This periodic incosistency leads to the interaction of the reflected waves with the incoming waves. Under specific circumstances, this interaction causes the partial or com- 
plete cancellation of wave propagation $[16,17]$. It can be easily shown that the frequency at which the band gap is observed depends on the length and the material/geometrical mismatch of the unit cell of the periodic structure. This leads to the need for prohibitively large dimensions to achieve low frequency band gaps. Therefore, researchers focused on local resonance [18], where a solid core material with relatively high density is usually preferred, suppressed by an elastically soft material, presenting band gaps near $400 \mathrm{~Hz}$. When this subwavelength inclusion/addition resonates, it exhibits behaviour that cancels the propagation of waves, giving rise to effective negative elastic constants or group velocities at certain frequency ranges which are significantly lower than those observed in Bragg scattering. Liu et al. [19] examined the transition between the two band gap production mechanisms and there has been research on coupling of the two mechanisms [20,21], where band gaps near $2 \mathrm{kHz}$ were achieved in low-stiffness structures. Recently an additional mechanism of band gap genera-

45 tions is gaining interest. This mechanism is called inertial amplification and has been proposed as band gap generator by Yilmaz et al. [22, 23]. In this approach inertial forces are enhanced between two points in a periodic cell of a structure. This way anti-resonance frequencies are generated, where the enhanced inertia cancels the elastic forces. This solution has been examined in various applications, where it offers wide low-frequency band gaps. In [24] Acar et al. produced numerical and experimental results examining the application of the inertial amplification mechanims in a two-dimensional solid structure. In the same scope Yuksel et al. [25] optimised the shape of phononic band gap structure implementing inertial amplification mechanims and produced wide band gap below the final result. Then, in $[26,27]$ researchers applied the inertial amplification mechanism on load-bearing structures without compromising their stiffnesses by adding the mechanism on the structure. Nevertheless, in both cases the application resulted in additional mass or compromised structural integrity. Hussein et

60 al. [16] produced an extensive review of developments in band gap technology. In this work the Bragg scattering method is examined as band gap generator. 
A sandwich structure is formed when two thin facings or skins are bonded on a lighter core that is used to keep them together and apart. This formation offers increased bending and torsional stiffness with minimal added mass, as lightweight core materials are used (eg. honeycomb, balsa wood and foam) [28]. The first application of lightweight structural sandwich parts was seen in aircrafts in the 1940s since they reduced the weight and increased the payload and flight distance [29]. Since then sandwich formations have been a major part of load bearing structures and applications can be found in many industries, such as civil transportation [30,31,32] and architecture [33]. Nowadays research is focused on examining the static behaviour of innovative solutions of sandwich formations, like a cylinder with orthogonal corrugated trusses [34], with orthogonal grids [35] or with beams with rapid prototyping cylinders as core [36]. A wide research is, also, focused on corrugated cores [37, 38, 39].

Taking advantage of the band gap mechanisms, researchers examined the possibility of having sandwich structures exhibiting stop bands. Ruzzene et al. $[40,41]$ examined theoretically the behaviour and control of sandwich beams and plates with auxetic core. It has been proven that photonic band gaps can be attained with periodic lattice core [42] and in [43,44] high-frequency band gaps are exhibited by sandwich phononic crystal plates. Local resonance mechanism has, also, been examined as band gap generator in sandwich structures [45], where Sharma et al. focused on the interaction and control of the band gap and how to tune the characteristics to achieve specific stop bands. In [46] Jiang et al. examined theoretically and experimentally a phononic crystal of periodic circular cavity sandwich plates attaining low-frequency $(\sim 500 \mathrm{~Hz})$ stop bands. The phononic crystal is made of steel, which excludes it from the group of lightweight structure, and it can handle axial loads. In [47] Badran et al. developed a lighter model to simulate the dynamic behaviour of sandwich structures. Guo et al. [48] examined theoretically the banded behaviour of a sandwich structure using two periodically alternating viscoelastic materials as core, achieving low-frequency $(\sim 100 \mathrm{~Hz})$ stop bands while compromising its load-bearing capabilities. Also, the effect of damping on the band gap was con- 
sidered and in [49] Sheng et al. manufactured the same structure to examine it experimentally. In $[50,51]$ the effect of the banded behaviour of a sandwich plate with locally resonant structure on the transmission of the sound is examined, proving that band gap offers significantly enhanced sound transmission loss. A thorough review of the current research on sandwich structures was produced by Birman and Kardomatea [52]. In this work two composite sandwich structures demonstrating band gap are examined, with skins made of carbon fibre and the core being PVC foam.

To the authors' knowledge, no research has been done on lightweight structural sandwich beams with band gaps lower than $5 \mathrm{kHz}$, where the highest sensitivity of the human ear lies [53], without additional mechanisms (hence mass) or significant compromise of structural integrity. Band gap mechanisms include high-displacement mechanisms or significant impedance mismatch which renders the design of a structural part very difficult and of quite complex geometries. In this work this specific problem is addressed, with the novelty of this paper being the easy-to-manufacture architecture of the two core designs of a lightweight sandwich beam which exhibit band gaps while not completely sacrificing load bearing ability of the structure. A finite element (FE) scheme coupled to PST is employed for computing the banded behaviour of the beams. Experimental analysis of the two schemes is conducted using a laser vibrometer and electrodynamic shaker setup. The results exhibit quite high correlation between numerical predictions and experimental measurements.

115

The paper is organised as follows: in Section 2 the methodology that is used during the numerical examination is described. In Section 3 the novel architectures of the two examined schemes are presented. The FE and experimental results along with the numerical ones are presented and discussed in Section 4 and finally in Section 5 the conclusions of the work are drawn. 


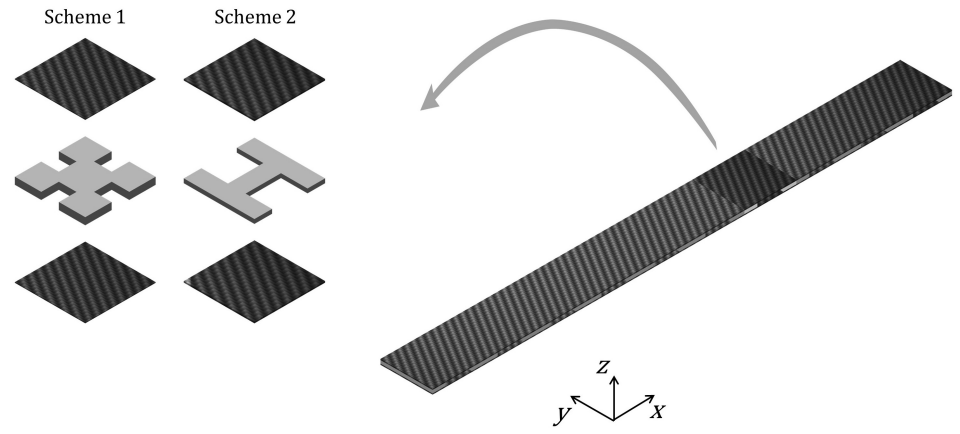

Figure 1: Caption of the modelled periodic structure. The core architectures that were examined are shown.

\section{Methodology}

\subsection{Periodic structure theory}

A generic structure with one-dimensional periodicity is considered. A unit cell is extracted from the structure (see Fig. 1) and its behaviour modelled with FE methods. A steady-state free harmonic vibration of frequency $\omega$ is considered in what follows and all response quantities are represented by complex amplitudes, so that

$$
\mathbf{q}(t)=\operatorname{Re}\left\{\mathbf{q} \mathrm{e}^{\mathrm{i} \omega t}\right\}
$$

where $t$ is time and the DOFs q can be partitioned into interior (I), left (L) and right (R) DOFs (see Fig. 2). According to Floquet's theorem, the equation that relates the displacements on the edges and sides of the modelled unit cell is the following [3]:

$$
\mathbf{q}_{R}=\mathrm{e}^{-\mathrm{i} \varepsilon_{x}} \mathbf{q}_{L}
$$

where the term $\varepsilon_{x}=k_{x} L_{x}$ is referred to as 'phase constant', $L_{x}$ is the periodic element's length in $x$ direction and $k_{x}$ is the wavenumber. The complete vector of the local DOFs can be ordered

$$
\mathbf{q}=\left[\begin{array}{lll}
\mathbf{q}_{I}^{T} & \mathbf{q}_{L}^{T} & \mathbf{q}_{R}^{T}
\end{array}\right]^{T}
$$




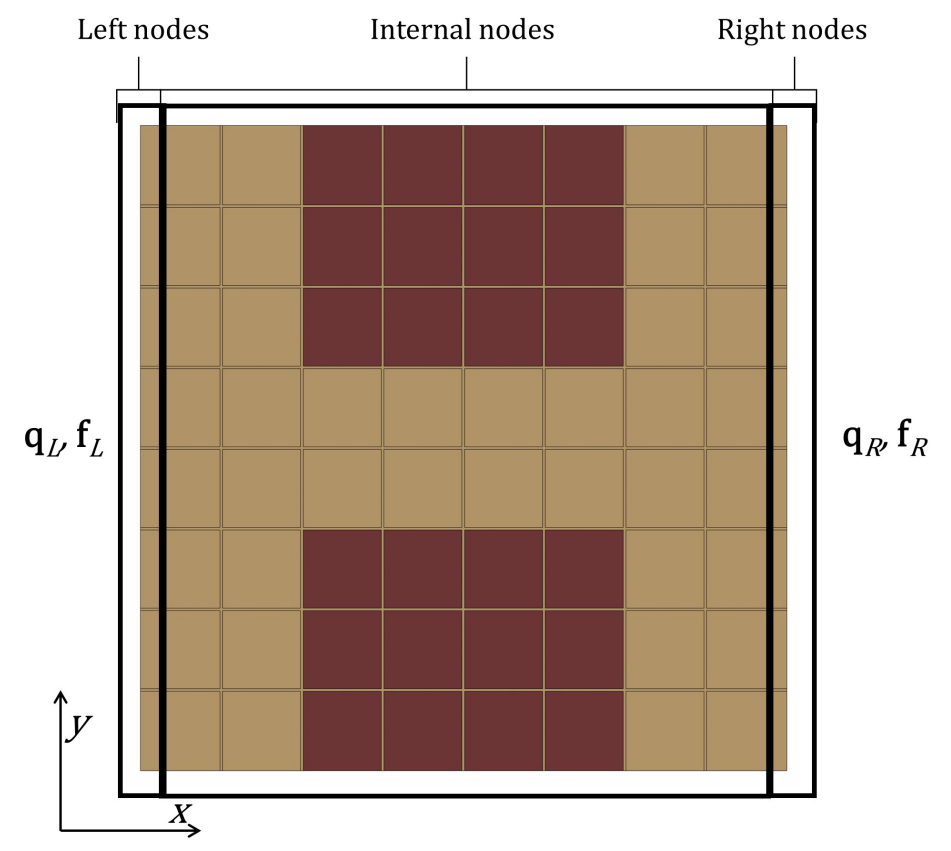

Figure 2: Caption of the one-dimensional unit cell. The degrees of freedom (DOFs) are grouped in left, internal and right ones during the numerical examination.

The equation of motion for the cell including damping [54] is given by

$$
\left[\mathbf{K}+\mathrm{i} \omega \mathbf{C}-\omega^{2} \mathbf{M}\right] \mathbf{q}=\mathbf{f}
$$

where $\mathbf{K}, \mathbf{M}$ and $\mathbf{C}$ are the stiffness, mass and damping matrices respectively and $\mathbf{f}$ is the vector of the nodal forces. Eq. (4) can be used when proportional damping is available [55], where damping is expressed as a linear combination of the mass and stiffness matrices, that is,

$$
\mathbf{C}=\alpha_{1} \mathbf{M}+\alpha_{2} \mathbf{K}
$$

where $\alpha_{1}$ and $\alpha_{2}$ are real scalars. In the case of structural damping [9, 54], the equation of motion becomes

$$
\left[\mathbf{K}+\mathrm{i} \mathbf{C}-\omega^{2} \mathbf{M}\right] \mathbf{q}=\mathbf{f}
$$


and the structural damping matrix $\mathbf{C}$ is given by

$$
\mathbf{C}=\sum_{i=1}^{n_{e}} \mathbf{K}_{i} \eta_{i}
$$

where $n_{e}$ is the number of the FEs of the unit cell, $\mathbf{K}_{i}$ is the stiffness matrix of the $i_{t h}$ element and $\eta_{i}$ is the loss factor of the corresponding element. Eq. (6) can be written as

$$
\left[\mathbf{K}(1+i \eta)-\omega^{2} \mathbf{M}\right] \mathbf{q}=\mathbf{f} .
$$

In this work, we do not focus on the accuracy of the model of damping, since the main aim of the wave propagation analysis is to examine the existence of the band gap. In order to write the propagation relation in Eq. (1) in matrix form, we consider transformation matrix $\mathbf{R}$, which is given in the following equation:

$$
\mathbf{q}=\left[\begin{array}{cc}
\mathbf{I} & \mathbf{0} \\
\mathbf{0} & \mathbf{I} \\
\mathbf{0} & \mathbf{I}^{-\mathrm{i} \varepsilon_{x}}
\end{array}\right] \mathbf{x}=\mathbf{R} \mathbf{x}
$$

In this way, we get

$$
\mathbf{q}=\mathbf{R} \mathbf{q}^{\prime}
$$

where

$$
\mathbf{q}^{\prime}=\mathbf{x}=\left[\begin{array}{ll}
\mathbf{q}_{I} & \mathbf{q}_{L}
\end{array}\right]^{T} .
$$

In the absence of external forces (free wave propagation), we have

$$
\mathbf{R}^{H} \mathbf{f}=\mathbf{0}
$$

where $\mathbf{R}^{H}$ denotes the complex conjugate (Hermitian) transpose of $\mathbf{R}$. The resulting homogenous equations in the reduced set of degrees of freedom are then given by

$$
\left[\mathbf{K}^{\prime}+\mathrm{i} \omega \mathbf{C}^{\prime}-\omega^{2} \mathbf{M}^{\prime}\right] \mathbf{q}=\mathbf{0}
$$

and

$$
\left[\mathbf{K}^{\prime}(1+\mathrm{i} \eta)-\omega^{2} \mathbf{M}^{\prime}\right] \mathbf{q}^{\prime}=\mathbf{0},
$$


where

$$
\mathbf{K}^{\prime}=\mathbf{R}^{H}\left(\varepsilon_{x}\right) \mathbf{K R}\left(\varepsilon_{x}\right), \quad \mathbf{M}^{\prime}=\mathbf{R}^{H}\left(\varepsilon_{x}\right) \mathbf{M R}\left(\varepsilon_{x}\right), \quad \mathbf{C}^{\prime}=\mathbf{R}^{H}\left(\varepsilon_{x}\right) \mathbf{C R}\left(\varepsilon_{x}\right) .
$$

When a set of phase constants $\varepsilon_{x}$ is specified, we get a quadratic eigenvalue problem for Eq. (13), the solution method of which is described elsewhere [56]. Eq. (14) gives a standard eigenvalue problem with eigenvalues $\lambda=\omega^{2}$ indicating 125 the frequencies at which a wave can propagate in the structure when a given phase is specified between the edges of the cell. It is noted that in this work, only the real part of the eigenvalues is examined since the target of the examination is the band gap behaviour.

The methodology described above is transferred in MATLAB and ANSYS environments through a master script generated specifically for this purpose which combines newly developped scripts with embedded existing tools [57]. The master script takes as input the geometry of the unit cell of the examined structure as produced in FE software and gives as output the wave propagation characteristics of the periodic structure (Figure 3). The steps are described in detail below:

- The geometry of the unit cell of the examined structure is produced in .txt file of ANSYS script language. This file also includes the material properties of the structure and these properties are assigned to each element of the cell to represent the actual structure.

- A simple and quick modal analysis is solved using ANSYS through the script so that mass, damping and stiffness matrices can be calculated. Using FE software to retrieve mass and stiffness matrices allows the user to simulate complex geometries.

- The matrices are retrieved by the script and moved to the folder-in-use.

- Then the matrices need to be converted to form MATLAB sparse matrices. For this step a freeware converter script was used [57] in order to convert the ANSYS matrices format (Harwell Boeing, HB) to MATLAB format. 
- After having the matrices in MATLAB format, the mathematical script of PST equations is run. This script can solve both one- and two-dimensional case studies.

- Finally, the wave propagation curve is calculated and plotted. In this step additional post-processing scripts can be implemented.

The script offers the chance to examine different structures quickly, since the only thing that the user needs to produce is the geometry file as all the rest is automated and included in the master script. Additionally, using FE software to retrieve mass and stiffness matrices allows the user to simulate complicated geometries. This way several schemes were examined before ending up to the ones presented in the next section.

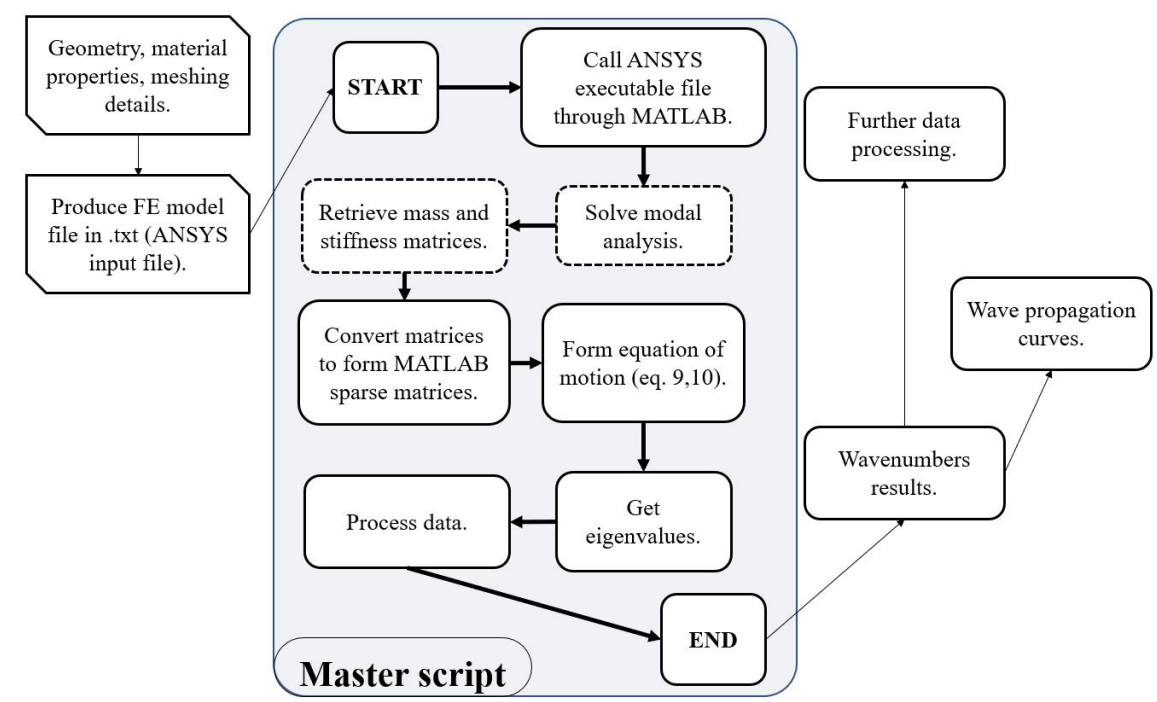

Figure 3: Flow diagram of the master script used to acquire wave propagation characteristics of the examined structures. The text boxes with dashed outline (- -) consist of ANSYS actions.

\section{Proposed core architecture}

The fundamental principle to save energy consumption and manufacturing cost is weight reduction. Additionally, the manufacturability of the structure 
must always be part of the design process in order to enable the industrilisation of the design. Having these two principles in mind two one-dimensional cases are developed and numerically and experimentally examined in this paper. Taking advantage of the core unconventional geometry, no additional mechanism is required avoiding extra mass. The work is based on lessons that were learnt in previous draft studies where a simple design of a sandwich beam with periodically hollow core acting as band gap generator was employed. The core-no core design was simulated and parametrically examined, where it presented banded behaviour in all the different alternative designs that were modelled. Nevertheless, the examined beam could not be considered as structural since there was no continuous part of core to handle the shear loading of the two skins during bending.
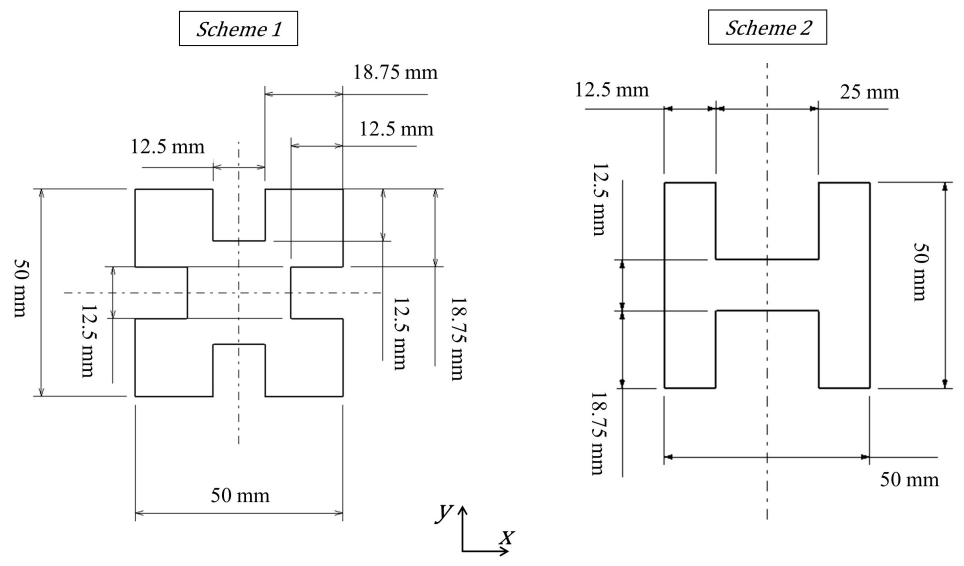

Figure 4: Geometry of the core of the scheme 1 and 2 unit cells.

Taking into consideration all the above, several alternatives were tested using the master script. All designs were conceived to combine core inconsistency in $\mathrm{x}$-axis and structural integrity. Symmetry along both $\mathrm{x}$ - and $\mathrm{y}$-axes was sought after in line with manufacturability and the examined solutions followed the dimensions of the commercially available materials. The schemes that are presented here have part of their cores continuous along $x$ axis, a characteristic 


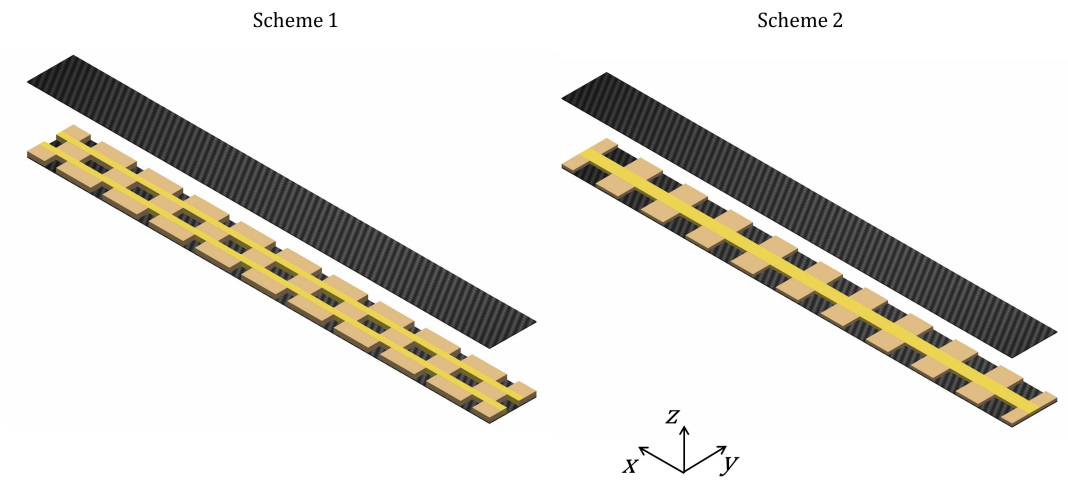

Figure 5: Skin and core of scheme 1 and 2 specimen.

Table 1: Material properties and Schemes characteristics.

\begin{tabular}{|c|c|c|}
\hline Scheme 1 & Scheme 2 & Both Schemes \\
\hline $0.5 \mathrm{~mm}$ Skin, $5 \mathrm{~mm}$ Core & $1 \mathrm{~mm}$ Skin, $3 \mathrm{~mm}$ Core & \\
\hline Cured Carbon Fibre & Cured Carbon Fibre & PVC Foam \\
\hline$\rho=1600 \mathrm{~kg} / \mathrm{m}^{3}$ & $\rho=1420 \mathrm{~kg} / \mathrm{m}^{3}$ & $\rho=100 \mathrm{~kg} / \mathrm{m}^{3}$ \\
\hline$E_{x}=50 \mathrm{GPa}$ & $E_{x}=55 \mathrm{GPa}$ & $E=75 \mathrm{MPa}$ \\
\hline$E_{y}=50 \mathrm{GPa}$ & $E_{y}=50 \mathrm{GPa}$ & $G=27.75 \mathrm{MPa}$ \\
\hline$E_{z}=20 \mathrm{GPa}$ & $E_{z}=20 \mathrm{GPa}$ & $\eta=4 \%$ \\
\hline$\nu_{x y}=0.4$ & $\nu_{x y}=0.4$ & \\
\hline$\nu_{y z}=0.4$ & $\nu_{y z}=0.4$ & \\
\hline$\nu_{x z}=0.25$ & $\nu_{x z}=0.25$ & \\
\hline$G_{x y}=1.2 \mathrm{GPa}$ & $G_{x y}=1.2 \mathrm{GPa}$ & \\
\hline$G_{y z}=1.2 \mathrm{GPa}$ & $G_{y z}=1.2 \mathrm{GPa}$ & \\
\hline$G_{x z}=3.6 \mathrm{GPa}$ & $G_{x z}=3.6 \mathrm{GPa}$ & \\
\hline$\eta=1 \%$ & $\eta=1 \%$ & \\
\hline
\end{tabular}


which renders them load bearing elements of a structure. The load bearing part of the core in bending loads is shown in different colour in Figure 5. To compare the load bearing capacity of the two schemes with the respective solid core beams, a 3-point bending beam simulation is considered using the FE models from Sec. 4.2. The setup can be seen in Fig. 6, where the beam is supported on both edges of the one side and a force $F=5 \mathrm{~N}$ is applied on the opposite edge on the middle point. The bending stiffness is then calculated using the equation:

$$
k=F / \delta z
$$

where $\delta z$ equals with the vertical displacement at the point of the force application. The results show that both schemes sacrifice a small percentage of the original bending stiffness (see Table 2).

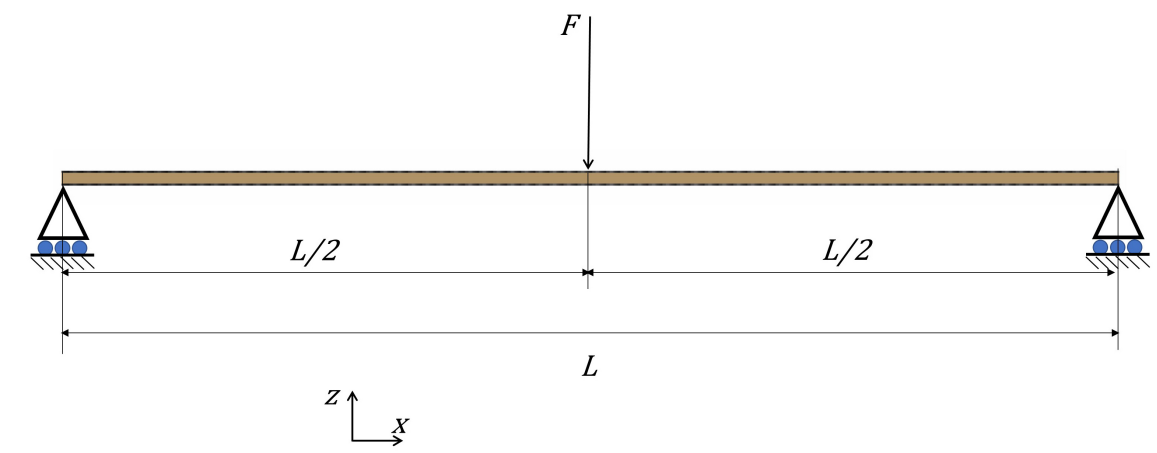

Figure 6: Schematic represantation of the cantilever beam simulation used to compare bending stiffness of the two schemes.

The core schemes can be easily manufactured using $2 \mathrm{D}$ automated cutting machine which puts no limitations to high-volume production. Both beams have carbon fibre as skin material and PVC foam as core material with different geometrical characteristics in both skin and core, see Table 1 . The geometry of both schemes' unit cell's core architecture is depicted in Fig. 4. Both schemes' geometry is symmetrical along $x$ and $y$ axis and scheme 1 is along the diagonal, too. The two architectures can be used to incorporate internal resonators to 
Table 2: Bending stiffness.

\begin{tabular}{c|cc|cc}
\hline \hline & \multicolumn{2}{|c|}{ Scheme 1 } & \multicolumn{2}{c}{ Scheme 2 } \\
\hline \hline & Proposed core & Solid core & Proposed core & Solid core \\
\hline$\delta \mathrm{z}$ & $0.479 \mathrm{~mm}$ & $0.417 \mathrm{~mm}$ & $0.335 \mathrm{~mm}$ & $0.275 \mathrm{~mm}$ \\
Bending stiffness & $10.44 \mathrm{kN} / \mathrm{m}$ & $11.99 \mathrm{kN} / \mathrm{m}$ & $14.93 \mathrm{kN} / \mathrm{m}$ & $18.18 \mathrm{kN} / \mathrm{m}$ \\
Difference & $12.94 \%$ & & $17.91 \%$ & \\
\hline
\end{tabular}

enhance the vibration annihilation behaviour. Additionally, another aspect that could be examined is the active control of vibrations using the necessary smart elements in the hollow parts of the cores. Both concepts are out of the scope of this work and are considered part of the future work.

\section{Experimental and numerical validation}

\subsection{Numerical analysis}

A FE model of each unit cell is built for both schemes, and the results of the wave propagation analysis are compared with unit cells of regular core sandwich beams with the same material properties (Table 1). Linear 8-node hexahedral ANSYS SOLID45 solid element is chosen for the segment's meshing, which comprises a 3D displacement field and three degrees of freedom per node (translations in $\mathrm{x}, \mathrm{y}$, and $\mathrm{z}$ directions). The number of the elements is decided according to the relevant convergence study results and wavelength requirement (6-10 elements per wavelength [5]) and so the model of scheme 1 unit cell is comprised of 176 elements, 48 of which are used to model the core, while the model of scheme 2 unit cell is comprised of 168 elements, 40 of which are used to model the core. In the case of the regular sandwich structure unit cell, the same number of elements are used for both of them (192 in total, 64 of which are used to model the core). In Fig. 7 and 8 the results of the wave propagation analysis are presented. As it can be seen in the graphs (shaded area), flexural band gap is observed at the frequency band $2271-2603 \mathrm{~Hz}$ for the scheme 1 and 


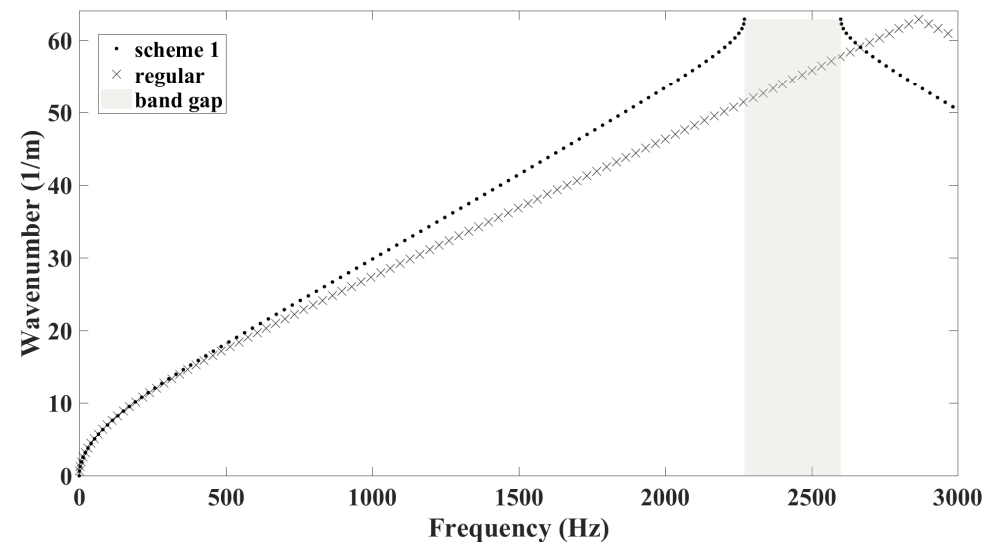

Figure 7: Flexural wave propagation of scheme 1 (.) and regular sandwich beam (x). The shaded area represents the bending band gap.

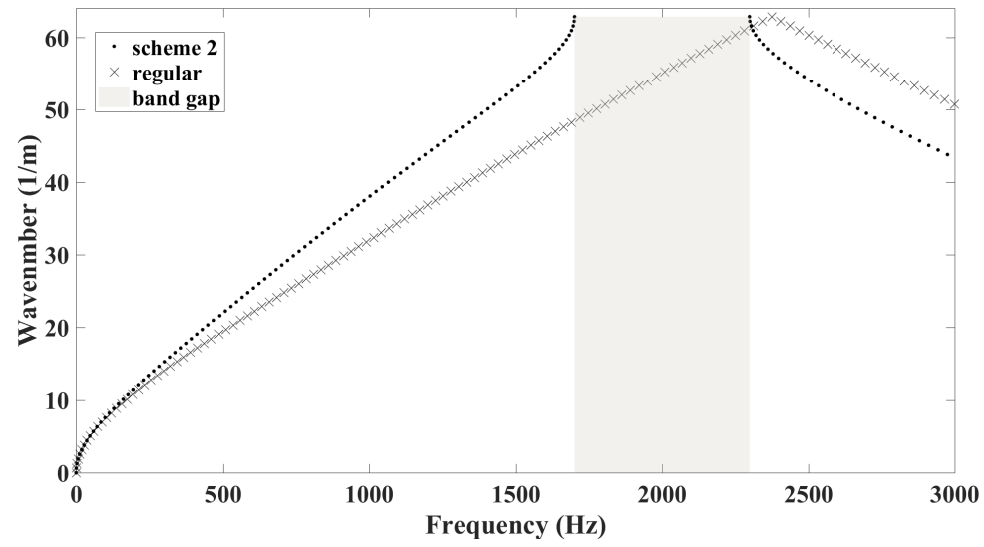

Figure 8: Flexural wave propagation of scheme $2($.$) and regular sandwich beam (x). The$ shaded area represents the bending band gap.

$1701-2299 \mathrm{~Hz}$ for the scheme 2. In order to examine the flexural behaviour of both schemes 10-cell beams were tested using both FE method and a laser vibrometer and electrodynamic shaker setup. The results are examined in the following paragraph. 


\subsection{FE and experimental results}

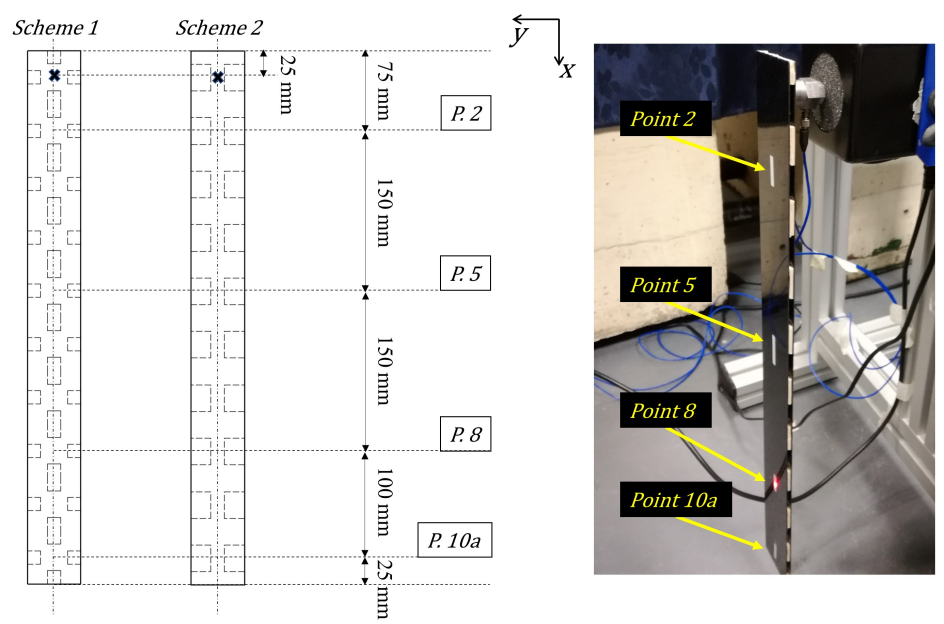

Figure 9: Left: The points that were examined in the experimental procedure and $\mathrm{FE}$ analyses. The point of excitation is marked as ' $\mathrm{X}$ '. Right: the points that were measured during the vibration experiment.

Following the same procedure as in Section 4.1 10-cell FE models of both schemes and regular sandwich beams with the same materials are produced. The same number of elements per unit cell was used, so the model of scheme 1 beam is comprised of 1760 elements, 480 of which are used to model the core, while the model of scheme 2 beam is comprised of 1680 elements, 400 of which are used to model the core. In the case of the regular sandwich beam structures, the same number of elements are used for both of them (1920 in total, 640 of which are used to model the core).

The core schemes were manufactured using two PVC foam sheets which were cut in the specific geometries, as seen in Fig. 10. After shaping the core schemes, the carbon fibre strips were cemented on both sides of them using a two-part epoxy adhesive (Permabond ET500). Concerning the experimental set up, an electrodynamic shaker (TBS K2007E1) is used to excite the specimen between $800 \mathrm{~Hz}$ and $3000 \mathrm{~Hz}$, with the signal produced and analysed by a Polytec VIBE-400 Junction Box using a fast Fourier transform (FFT). A single point laser 

be seen in Fig. 9, the points' number is indicative of the unit cell's repetition. The experimental setup employed in this work has already been proven in [58].

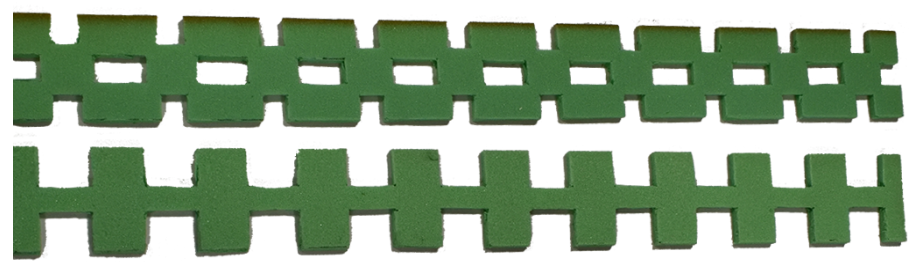

Figure 10: The two core schemes as were manufactured. The cores were then cemented on cured carbon fibre sheets.

In Fig. 11 and 12 the FEA and experimental results of all the measured points of both schemes 1 and 2 are presented. Firstly and most importantly, it can be observed that in both cases experimental and FEA results correlate, with only some anomalies for the point 2 graphs. Figures 13 and 14 exhibit the correlation of the peaks of the experimental and FEA curves at point 10, where the local divergence is less than $3 \%$. Secondly, both schemes 1 and 2 experiments demonstrate banded behaviour, as predicted in the numerical results in 4.1. The stop band is quite clear in the predicted frequency bands, and the vibration cancellation becomes more apparent as the points' number increase. This happens because as the wave propagates through the unit cells, it interacts with the reflective ones, which in the specific band gap frequency weaken it. Therefore, the wave passes weakened through each unit cell and 


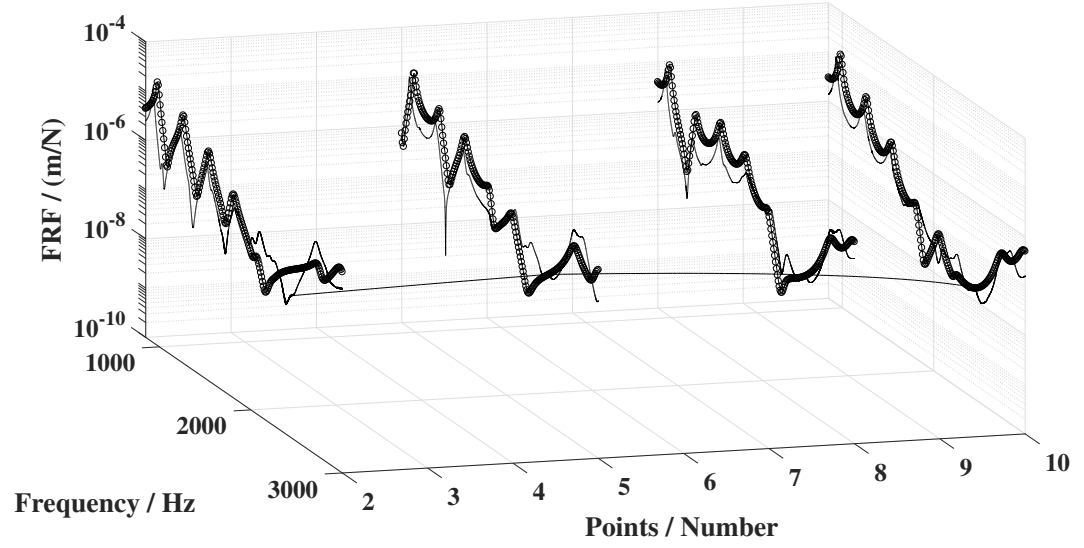

Figure 11: FRF of FEA (-o) and experimental results (-) of all the measured points of scheme 1. The points of the line connecting the FEA graphs consists of the middle value of the band gap.

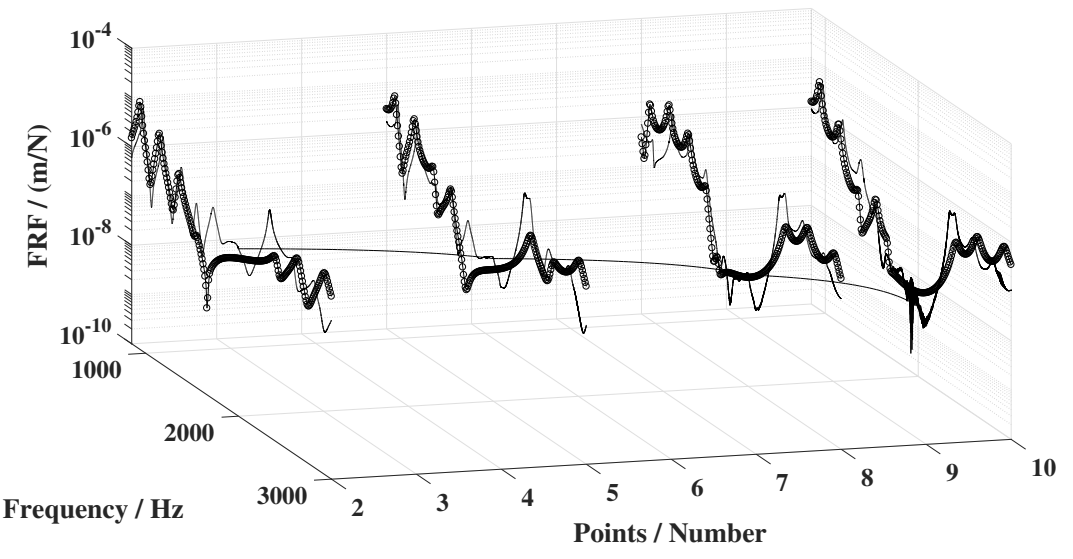

Figure 12: FRF of FEA (-o) and experimental results (-) of all the measured points of scheme 2. The points of the line connecting the FEA graphs consists of the middle value of the band gap.

the amplitude loss adds up as it goes through the beam. Eventually it gets significantly weakened which is translated in annihilation of the beam vibration. This observation coincides with the theory of band gap on periodic structures 


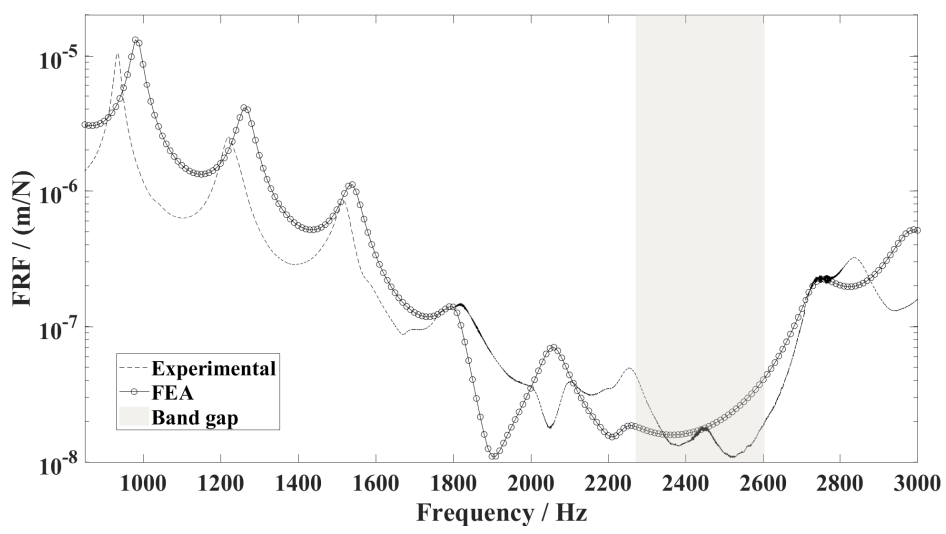

Figure 13: Scheme 1 experimental (-) and FEA (-o) results of point 10 displacement.

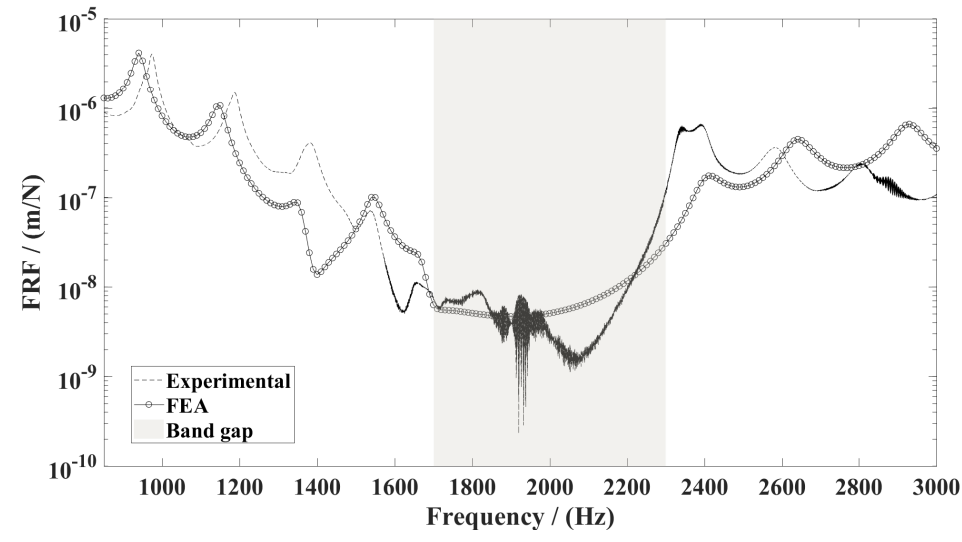

Figure 14: Scheme 2 experimental (-) and FEA (-o) results of point 10 displacement.
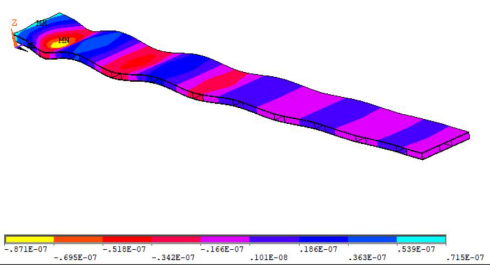

Figure 15: FEA of scheme 1 at $2430 \mathrm{~Hz}$ Figure 16: FEA of scheme 2 at $2000 \mathrm{~Hz}$ (displacements in meters).
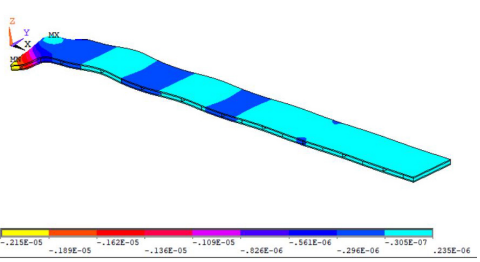

(displacements in meters). 
and it has been discussed in existing literature [58, 59, 60]. In Fig. 15 and 16 the displacement profiles of schemes 1 and 2, respectively, are presented, where the aforementioned observation is graphically represented. This behaviour must be taken into consideration during the design of the application of the banded structure so that to achieve the optimum vibration isolation. Another issue that must be considered is the boundary conditions of the structure which will probably alter the banded behaviour. Furthermore, further work could concern the incorporation of local resonant structures in the parts of the core that are hollow, employing both band gap mechanisms.

\section{Concluding remarks}

In this paper we present novel core architectures able to induce wave stop bands while not being detrimental for structural bending stiffness. The two designs are developed following weight reduction and manufacturability criteria to produce a low-cost and profitable solution. The main conclusions that are drawn are listed below:

- Firstly the numerical results of the wave propagation of the two core schemes demonstrate that both of the examined beams exhibit stop band of the flexural wave below $5000 \mathrm{~Hz}$. This low frequency stop band has not been observed in load-bearing structures without any additional internal resonance or inertial amplification mechanism.

- Secondly, quite high agreement is observed in the FE analysis and experimental results. This agreement validates the banded behaviour of the proposed core schemes.

- Thirdly, a master script is produced which gives the ability to the engineer to examine the banded behaviour of a complicated periodic structure quickly, since PST is used which is more time efficient than full FEA. Additionally, the master script offers all the needed calculations in one automated procedure. 
The two schemes, hence, consist of a load bearing solution of passively annihilating vibrations in specific frequency bands.

\section{References}

[1] G. Floquet, Sur les équations différentielles linéaires à coefficients périodiques, in: Annales scientifiques de l'École normale supérieure, Vol. 12, 1883, pp. 47-88.

[2] L. Rayleigh, XVII. On the maintenance of vibrations by forces of double frequency, and on the propagation of waves through a medium endowed with a periodic structure, The London, Edinburgh, and Dublin Philosophical Magazine and Journal of Science 24 (147) (1887) 145-159. doi: $10.1080 / 14786448708628074$.

[3] D. J. Mead, A general theory of harmonic wave propagation in linear periodic systems with multiple coupling, Journal of Sound and Vibration 27 (2) (1973) 235-260. doi:10.1016/0022-460X (73) 90064-3.

[4] D. M. Mead, Wave propagation in continuous periodic structures: research contributions from Southampton, 1964-1995, Journal of sound and vibration 190 (3) (1996) 495-524. doi:10.1006/jsvi.1996.0076.

[5] B. R. Mace, E. Manconi, Modelling wave propagation in two-dimensional structures using finite element analysis, Journal of Sound and Vibration 318 (4) (2008) 884-902. doi:10.1016/j.jsv.2008.04.039.

[6] V. Cotoni, R. S. Langley, P. J. Shorter, A statistical energy analysis subsystem formulation using finite element and periodic structure theory, Journal of Sound and Vibration 318 (4) (2008) 1077-1108. doi: $10.1016 / j \cdot j s v \cdot 2008.04 .058$.

[7] R. S. Langley, N. S. Bardell, P. M. Loasby, The optimal design of nearperiodic structures to minimize vibration transmission and stress levels, 
Journal of Sound and Vibration 207 (5) (1997) 627-646. doi:10.1006/ jsvi.1997.1116.

[8] L. Brillouin, Wave propagation in periodic structures: electric filters and crystal lattices, McGrawHil, 1946.

[9] E. Manconi, B. R. Mace, Estimation of the loss factor of viscoelastic laminated panels from finite element analysis, Journal of Sound and Vibration 329 (19) (2010) 3928-3939. doi:10.1016/j.jsv.2010.04.014.

[10] D. Chronopoulos, B. Troclet, O. Bareille, M. Ichchou, Modeling the response of composite panels by a dynamic stiffness approach, Composite Structures 96 (2013) 111-120. doi:10.1016/j . compstruct.2012.08.047.

[11] T. Ampatzidis, D. Chronopoulos, Acoustic transmission properties of pressurised and pre-stressed composite structures, Composite Structures 152 (2016) 900-912. doi:10.1016/j.compstruct.2016.06.027.

[12] I. Antoniadis, D. Chronopoulos, V. Spitas, D. Koulocheris, Hyper-damping properties of a stiff and stable linear oscillator with a negative stiffness element, Journal of Sound and Vibration 346 (2015) 37-52. doi:10.1016/ j.jsv.2015.02.028.

[13] D. Chronopoulos, I. Antoniadis, T. Ampatzidis, Enhanced acoustic insulation properties of composite metamaterials having embedded negative stiffness inclusions, Extreme Mechanics Letters 12. doi:10.1016/j.eml. 2016.10 .012 .

[14] L. Van Belle, C. Claeys, E. Deckers, W. Desmet, On the impact of damping on the dispersion curves of a locally resonant metamaterial: Modelling and experimental validation, Journal of Sound and Vibration 409 (2017) 1-23. doi:10.1016/j.jsv.2017.07.045.

[15] C. Claeys, E. Deckers, B. Pluymers, W. Desmet, A lightweight vibroacoustic metamaterial demonstrator: Numerical and experimental inves- 
tigation, Mechanical Systems and Signal Processing 70 (2016) 853-880. doi:10.1016/j.ymssp.2015.08.029.

[16] M. I. Hussein, M. J. Leamy, M. Ruzzene, Dynamics of phononic materials and structures: Historical origins, recent progress, and future outlook, Applied Mechanics Reviews 66 (4) (2014) 40802. doi:10.1115/1.4026911.

[17] M. I. Hussein, G. M. Hulbert, R. A. Scott, Dispersive elastodynamics of 1D banded materials and structures: analysis, Journal of sound and vibration 289 (4) (2006) 779-806. doi:10.1016/j.jsv.2005.02.030.

[18] Z. Liu, X. Zhang, Y. Mao, Y. Y. Zhu, Z. Yang, C. T. Chan, P. Sheng, Locally resonant sonic materials, Science 289 (5485) (2000) 1734-1736. doi:10.1126/science.289.5485.1734.

[19] L. Liu, M. I. Hussein, Wave motion in periodic flexural beams and characterization of the transition between Bragg scattering and local resonance, Journal of Applied Mechanics 79 (1) (2012) 11003. doi:10.1115/ 1.4004592 .

[20] J. S. Chen, C. T. Sun, Wave propagation in sandwich structures with resonators and periodic cores, Journal of Sandwich Structures \& Materials 15 (3) (2013) 359-374. doi:10.1177/1099636212468738.

[21] K. H. Matlack, A. Bauhofer, S. Krödel, A. Palermo, C. Daraio, Composite 3D-printed metastructures for low-frequency and broadband vibration absorption, Proceedings of the National Academy of Sciences (2016) 201600171doi:10.1073/pnas.1600171113.

[22] C. Yilmaz, N. Kikuchi, Analysis and design of passive low-pass filter-type vibration isolators considering stiffness and mass limitations, Journal of Sound and Vibration 293 (1-2) (2006) 171-195. doi:10 . 1016/J . JSV . 2005. 09.016. 
[23] C. Yilmaz, G. Hulbert, Theory of phononic gaps induced by inertial amplification in finite structures, Physics Letters A 374 (34) (2010) 3576-3584. doi:10.1016/J . PHYSLETA . 2010.07.001.

[24] G. Acar, C. Yilmaz, Experimental and numerical evidence for the existence of wide and deep phononic gaps induced by inertial amplification in two-dimensional solid structures, Journal of Sound and Vibration 332 (24) (2013) 6389-6404. doi:10.1016/J. JSV . 2013 .06.022.

[25] O. Yuksel, C. Yilmaz, Shape optimization of phononic band gap structures incorporating inertial amplification mechanisms, Journal of Sound and Vibration 355 (2015) 232-245. doi:10.1016/J. JSV.2015.06.016.

[26] N. M. M. Frandsen, O. R. Bilal, J. S. Jensen, M. I. Hussein, Inertial amplification of continuous structures: Large band gaps from small masses, Journal of Applied Physics 119 (12) (2016) 124902. arXiv:1512.09182, doi:10.1063/1.4944429.

[27] J. Li, S. Li, Generating ultra wide low-frequency gap for transverse wave isolation via inertial amplification effects, Physics Letters A 382 (5) (2018) 241-247. doi:10.1016/J . PHYSLETA . 2017.11.023.

[28] C. Zweben, Composite Materials, CRC Press, 2007. arXiv:arXiv:1011. 1669v3, doi:10.1002/9780470172551.ch12.

[29] D. B. Miracle, S. L. Donaldson, S. D. Henry, W. W. Scott, J. J. Brown Kelly Ferjutz Edward Kubel, J. Heather Lampman Elizabeth Marquard Beverly Musgrove Mary Jane Riddlebaugh Juli Williamson, ASM Handbook Volume 21 Composites Prepared under the direction of the ASM International Handbook Committee, Tech. rep. (2001).

[30] L. Torre, J. M. Kenny, Impact testing and simulation of composite sandwich structures for civil transportation, Composite structures 50 (3) (2000) 257267. doi:10.1016/S0263-8223(00)00101-X. 
[31] H. Ning, G. M. Janowski, U. K. Vaidya, G. Husman, Thermoplastic sandwich structure design and manufacturing for the body panel of mass transit vehicle, Composite Structures 80 (1) (2007) 82-91. doi:10.1016/j. compstruct.2006.04.090.

[32] A. S. Herrmann, P. C. Zahlen, I. Zuardy, Sandwich structures technology in commercial aviation, in: Sandwich structures 7: Advancing with sandwich structures and materials, Springer, 2005, pp. 13-26. doi: 10.1007/1-4020-3848-8_2.

[33] J. Hegger, M. Horstmann, Light-weight TRC sandwich building envelopes, in: Proceedings of the International Conference Excellence in Concrete Construction through Innovation, 2008, pp. 187-194.

[34] W. Li, F. Sun, P. Wang, H. Fan, D. Fang, A novel carbon fiber reinforced lattice truss sandwich cylinder: fabrication and experiments, Composites Part A: Applied Science and Manufacturing 81 (2016) 313-322. doi:10. 1016/j.compositesa.2015.11.034.

[35] S. Jiang, F. Sun, H. Fan, D. Fang, Fabrication and testing of composite orthogrid sandwich cylinder, Composites Science and Technology 142 (2017) 171-179. doi:10.1016/j.compscitech.2017.02.009.

[36] J. V. Nygaard, A. Lyckegaard, Sandwich beam with a periodical and graded core manufactured using rapid prototyping, Journal of Sandwich Structures \& Materials 9 (4) (2007) 365-376. doi:10.1177/1099636207071609.

[37] B. Han, K.-K. Qin, B. Yu, Q.-C. Zhang, C.-Q. Chen, T. J. Lu, Design optimization of foam-reinforced corrugated sandwich beams, Composite Structures 130 (2015) 51-62. doi:10.1016/j. compstruct.2015.04.022.

[38] R. Biagi, H. Bart-Smith, In-plane column response of metallic corrugated core sandwich panels, International Journal of Solids and Structures 49 (26) (2012) 3901-3914. doi:10.1016/j.ijsolstr.2012.08.015. 
[39] M. R. M. Rejab, W. J. Cantwell, The mechanical behaviour of corrugatedcore sandwich panels, Composites Part B: Engineering 47 (2013) 267-277. doi:10.1016/j.compositesb.2012.10.031.

[40] M. Ruzzene, F. Scarpa, Control of wave propagation in sandwich beams with auxetic core, Journal of intelligent material systems and structures 14 (7) (2003) 443-453. doi:10.1177/1045389x03035515.

[41] M. Ruzzene, P. Tsopelas, Control of wave propagation in sandwich plate rows with periodic honeycomb core, Journal of engineering mechanics 129 (9) (2003) 975-986. doi:10.1061/(ASCE) 0733-9399(2003)129: $9(975)$.

[42] Y. Pang, B. Gao, A new sandwich structure of photonic bandgap, in: Microwave Symposium Digest, 2002 IEEE MTT-S International, Vol. 2, IEEE, 2002, pp. 1157-1160. doi:10.1109/MWSYM.2002.1011853.

[43] Y. Cheng, X. J. Liu, D. J. Wu, Band structures of phononic-crystal plates in the form of a sandwich-layered structure, The Journal of the Acoustical Society of America 130 (5) (2011) 2738-2745. doi:10.1121/1.3641365.

[44] X.-f. Liu, Y.-f. Wang, Y.-s. Wang, Complete band gaps in sandwich phononic crystal plates, in: Piezoelectricity, Acoustic Waves and Device Applications (SPAWDA), 2011 Symposium on, IEEE, 2011, pp. 433-436. doi:10.1109/SPAWDA. 2011.6167281.

[45] B. Sharma, C.-T. Sun, Local resonance and Bragg bandgaps in sandwich beams containing periodically inserted resonators, Journal of Sound and Vibration 364 (2016) 133-146. doi:10.1016/j.jsv.2015.11.019.

[46] S. Jiang, H. Chen, L. Dai, H. Hu, V. Laude, Multiple low-frequency broad band gaps generated by a phononic crystal of periodic circular cavity sandwich plates, Composite Structures 176 (2017) 294-303. doi: $10.1016 / j$. compstruct. 2017.05 .048 . 
[47] H. Badran, M. Tawfik, H. Negm, Vibration characteristics of periodic sandwich beams, in: Proceedings of the 13th International Conference on Applied Mechanics and Mechanical Engineering, 2008. doi:10.13140/2.1. 5121.3122.

[48] Z. Guo, M. Sheng, J. Pan, Flexural wave attenuation in a sandwich beam with viscoelastic periodic cores, Journal of Sound and Vibration 400 (2017) 227-247. doi:10.1016/j.jsv.2017.04.016.

${ }_{445}$ [49] M. Sheng, Z. Guo, Q. Qin, Y. He, Vibration characteristics of a sandwich plate with viscoelastic periodic cores, Composite Structuresdoi:10.1016/ j.compstruct.2018.07.110.

[50] Y. Song, L. Feng, J. Wen, D. Yu, X. Wen, Reduction of the sound transmission of a periodic sandwich plate using the stop band concept, Composite Structures 128 (2015) 428-436. doi:10.1016/J. COMPSTRUCT. 2015. 02.053.

[51] Z. Liu, R. Rumpler, L. Feng, Broadband locally resonant metamaterial sandwich plate for improved noise insulation in the coincidence region, Composite Structures 200 (2018) 165-172. doi:10.1016/J.COMPSTRUCT. 2018.05 .033$.

[52] V. Birman, G. A. Kardomatea, Review of current trends in research and applications of sandwich structures, Composites Part B: Engineeringdoi: $10.1016 / j$. compositesb. 2018.01.027.

[53] S. A. Gelfand, Essentials of audiology, Thieme, 2009.

[54] S. Neumark, Concept of complex stiffness applied to problems of oscillations with viscous and hysteretic damping, HM Stationery Office, 1962.

[55] S. Adhikari, Damping modelling using generalized proportional damping, Journal of Sound and Vibration 293 (1-2) (2006) 156-170. doi:10.1016/ j.jsv.2005.09.034. 
[56] F. Tisseur, K. Meerbergen, The quadratic eigenvalue problem, SIAM review 43 (2) (2001) 235-286.

[57] I. Duff, R. Grimes, J. Lewis, User's Guide for the Harwell-Boeing Sparse Matrix Collection, Tech. rep. (1992).

[58] T. Ampatzidis, R. K. Leach, C. J. Tuck, D. Chronopoulos, Band gap behaviour of optimal one-dimensional composite structures with an additive manufactured stiffener, Composites Part B: Engineering 153 (2018) 26-35. doi:10.1016/j.compositesb.2018.07.012.

[59] J. S. Jensen, Phononic band gaps and vibrations in one-and twodimensional mass-spring structures, Journal of Sound and Vibration 266 (5) (2003) 1053-1078. doi:10.1016/S0022-460X (02) 01629-2.

[60] P. G. Domadiya, E. Manconi, M. Vanali, L. V. Andersen, A. Ricci, Numerical and experimental investigation of stop-bands in finite and infinite periodic one-dimensional structures, Journal of Vibration and Control 22 (4) (2016) 920-931. doi:10.1177/1077546314537863. 